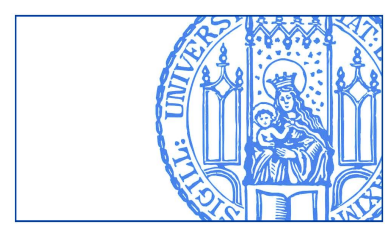

Schlicht, Ekkehart:

The Moving Equilibrium Theorem Again

Munich Discussion Paper No. 1997-51

Department of Economics

University of Munich

Volkswirtschaftliche Fakultät

Ludwig-Maximilians-Universität München

Online at https://doi.org/10.5282/ubm/epub.39121 


\title{
The Moving Equilibrium Theorem Again
}

\author{
Ekkehart Schlicht*
}

Electronic reprint from Economic Modelling 14 (1997) 271-278, with some corrections.

\begin{abstract}
The analysis of multivariate dynamical models can sometimes be considerably simplified by the assumption that one or several variables move infinitely fast to their equilibrium values. The method is known as the 'moving equilibrium method'. Various dynamical theories that build on equilibrated markets presuppose the validity of this method. The Moving Equilibrium Theorem states that such an analytic procedure leads to correct conclusions if the movement of the fast variables is indeed sufficiently fast. The purpose of this paper is to present a simple direct proof of the theorem for the linear case.
\end{abstract}

JEL classification: B41; C6

Keywords: Moving equilibrium; Dynamical systems; Temporary equilibrium

${ }^{*}$ Professor emeritus of Economics, Department of Economics, Ludwig-MaximiliansUniversität München, German, email: schlicht@lmu.de. 


\section{Introduction}

The analysis of multivariate dynamical models can sometimes be considerably simplified by the assumption that one or several variables move infinitely fast to their equilibrium values. The method is known as the 'moving equilibrium method' (Lotka 1957, Ch. 2; Samuelson, 1947, 321-323). In a less formal way it is underlying Marshall's (1920) distinction between long and short periods and temporary equilibria. It may even be said, and will be outlined later, that partial equilibrium economics rests on the premise that the moving equilibrium method delivers appropriate results.

However, Eckalbar (1985) has presented a set of examples that led him to conclude that the method is in general not valid and that "simplified systems behave like true systems only in highly unlikely cases" (p. 3). This would indeed be unfortunate, since it would cast important parts of partial equilibrium theory in doubt; but fortunately this view is much too pessimistic. The purpose of this paper is to present a new proof of the "Moving Equilibrium Theorem" which asserts that the moving equilibrium method is indeed a useful tool for analyzing dynamical systems in a simplified way. Some further observations and comments are added with the intention of indicating the relevance of the theorem for economic analysis.

\section{The moving equilibrium method}

Consider a differential equation system in two vectors of variables $x$ and $y$ :

$$
\dot{x}=f(x, y), \quad \dot{y}=g(x, y)
$$

where $x$ is the "fast" and $y$ the "slow" variable. Assume that the fast system, $f$, is stable and that for any given and fixed $y$, the fast vector $x$ tends to its equilibrium:

$$
x=X(y) \text {, with } f(X(y), y)=0 .
$$

If $y$ changes over time, then $x=X(y(t))$ gives the corresponding moving equilibrium of $x$. In analyzing the movement of the slow variable, the fast variable $x$ can be approximated by its equilibrium value $X$ in the equation describing the movement of the slow variable $y$. This gives rise to the following equations:

$$
\dot{Y}=g(X(Y), Y), \quad X=X(Y)
$$


which describe the movement of the variables $X$ and $Y$ that approximate $x$ and $y$. System (3) is thus the moving equilibrium approximation of the system (1). Since system (3) may be easier to analyze than system (1), the question arises: Under what circumstances does the moving equilibrium method lead to correct conclusions? The following section presents a theorem asserting the validity of the method in the linear (or local) case.

\section{The Moving Equilibrium Theorem}

Consider the following linear dynamical system

$$
\begin{aligned}
& \dot{x}=\alpha(A \cdot x+B \cdot y) \\
& \dot{y}=C \cdot x+D \cdot y
\end{aligned}
$$

where $x \in \mathbb{R}^{m}$ is the vector of "fast" state variables, $y \in \mathbb{R}^{n}$ is the vector of "slow"state variables and $\alpha>0$ is the speed of the fast variable. The dynamic behavior of the system is fully characterized by the following Jacobian

$$
J(\alpha)=\left(\begin{array}{cc}
\alpha A & \alpha B \\
C & D
\end{array}\right)
$$

The idea of the moving equilibrium method is to approximate the system (4), (5) for large $\alpha$ by another system that assumes that the fast variables $x$ have reached their equilibrium. Intuitively, this makes sense if the matrix $A$ describing the behavior of the fast variables $x$ for any given state of slow variables $y$ is stable (i.e. has only eigenvalues with negative real parts) and the speed $\alpha$ is sufficiently fast.

We assume therefore that $A$ is stable and hence non-singular. Given the non-singularity of $A$, the equilibrium of the fast variables is given by

$$
X=-A^{-1} B y \text {. }
$$

If this is inserted into (5), then another differential equation for the slow variables $y$ is obtained. This is, however, only an approximation, and we denote the approximation for $y$ obtained in this way by $Y$ :

$$
\dot{Y}=\left(D-C A^{-1} B\right) Y \text {. }
$$

The following theorem states that the stability of the fast system (4) for given $y$ and stability of the reduced system (8) imply stability of the true system (4), (5) if $\alpha$ is sufficiently large. 
Moving Equilibrium Theorem (Schlicht 1978, 1985, 39-45). If $A$ and $\left(D-C A^{-1} B\right)$ are stable and $\alpha$ is sufficiently large, then $J(\alpha)$ is stable.

Proof.

(1) Non-singularity of $J(\alpha)$. Since $A$ is non-singular, $J(\alpha)$ can be written as a product of two non-singular matrices:

$$
J(\alpha)=\left(\begin{array}{cc}
I & 0 \\
C(\alpha A)^{-1} & I
\end{array}\right)\left(\begin{array}{cc}
\alpha A & \alpha B \\
0 & D-C(\alpha A)^{-1} \alpha B
\end{array}\right) .
$$

Hence $J(\alpha)$ is non-singular for any $\alpha$.

(2) Classification of eigenvalues. Denote the spectrum of $J(\alpha)$ by

$$
\Lambda(\alpha)=\left(\lambda_{1}(\alpha), \lambda_{2}(\alpha), \ldots, \lambda_{N}(\alpha)\right) \text {, with } N=m+n .
$$

The eigenvalues $\lambda_{i}(\alpha)$ are continuous functions of $\alpha$. Consider the spectrum for $\alpha \rightarrow \infty$. Assume, without loss of generality, that the first $k$ eigenvalues remain bounded for $\alpha \rightarrow \infty$ and the remaining $N-k$ are unbounded, with $k \geq 0$ and $k \leq N$.

(3) Bounded eigenvalues. Consider any eigenvalue $\lambda_{i}(\alpha)$ that remains bounded for $\alpha \rightarrow \infty$, i.e. $i \leq k$. It satisfies the characteristic equation

$$
\operatorname{det}\left(J(\alpha)-\lambda_{i}(\alpha) I\right)=\operatorname{det}\left(\begin{array}{cc}
\alpha A-\lambda_{i}(\alpha) I & \alpha B \\
C & D-\lambda_{i}(\alpha) I
\end{array}\right)=0
$$

for all $\alpha$. Consider the matrix

$$
\left(\alpha A-\lambda_{i}(\alpha) I\right)
$$

For large $\alpha$ and bounded $\lambda_{i}(\alpha)$, this matrix will be non-singular, and we can use a decomposition similar to (9) to write the characteristic equation (11) as

$$
\operatorname{det}\left(\alpha A-\lambda_{i}(\alpha) I\right) \operatorname{det}\left(D-C\left(\alpha A-\lambda_{i}(\alpha) I\right)^{-1} \alpha B-\lambda_{i}(\alpha) I\right)=0
$$

This implies

$$
\operatorname{det}\left(D-C\left(A-\frac{\lambda_{i}(\alpha)}{\alpha} I\right)^{-1} B-\lambda_{i}(\alpha) I\right)=0
$$

As $\frac{\lambda_{i}(\alpha)}{\alpha}$ tends to zero for $\alpha \rightarrow \infty, \lambda_{i}(\alpha)$ must tend to an eigenvalue of $\left(D-C A^{-1} B\right)$. This matrix has been assumed to be stable. Thus, $\lambda_{i}(\alpha)$ must have a negative real part for sufficiently large $\alpha$. 
(4) Unbounded eigenvalues. Consider now an eigenvalue $\lambda_{i}(\alpha)$ that grows in size in an unbounded way for $\alpha \rightarrow \infty$, i.e. $i>k$. For large $\alpha, \lambda_{i}(\alpha)$ will be large and $\left(D-\lambda_{i}(\alpha) I\right)$ will be non-singular. The characteristic equation of (6) is now equivalent to

$$
\operatorname{det}\left(D-\lambda_{i}(\alpha) I\right) \operatorname{det}\left(A-\lambda_{i}^{-1}(\alpha) B\left(\lambda_{i}^{-1}(\alpha) D-I\right)^{1} C-\frac{1}{\alpha} \lambda_{i}^{-1}(\alpha) I\right)=0,
$$

with

$$
\lambda_{i}^{-1}(\alpha)=\frac{\operatorname{Re}\left(\lambda_{i}(\alpha)\right)-i \cdot \operatorname{Im}\left(\lambda_{i}(\alpha)\right)}{\left(\operatorname{Re}\left(\lambda_{i}(\alpha)\right)\right)^{2}+\left(\operatorname{Im}\left(\lambda_{i}(\alpha)\right)\right)^{2}}
$$

For $\lambda_{i}(\alpha)$ and $\alpha$ large, $\lambda_{i}^{-1}(\alpha) D$ tends to zero and the term $-\left(\lambda_{i}^{-1}(\alpha) D-I\right)$ approaches the identity matrix. Hence $\left(A-\lambda_{i}^{-1}(\alpha) B\left(\lambda_{i}^{-1}(\alpha) D-I\right)^{-1} C\right)$ is dominated by $A$ and $\frac{1}{\alpha} \lambda_{i}^{-1}(\alpha)$ must approach an eigenvalue of $A$. In particular, the expression

$$
\frac{1}{\alpha} \operatorname{Re}\left(\lambda_{i}(\alpha)\right)
$$

must approach the real part of an eigenvalue of $A$. Since $A$ has been assumed stable, this must be negative, and $\operatorname{Re}\left(\lambda_{i}(\alpha)\right) »$ must be negative as well. Q.E.D.

\section{On the Informal Use of the Moving Equilibrium Method in Economics}

The Moving Equilibrium Theorem is useful in offering a convenient analytical shortcut, and it is invoked, in this sense, quite frequently in less formal discussions. The following statement by Lindbeck $(1976,137)$ illustrates this:

One conceivable simplification of such macro analysis is to exploit the different speeds of adjustment between markets, which may make it possible to operate with recursive relations between various blocks in the macro model. For instance, based on the assumption that the market for financial assets clears rapidly, whereas the market for commodity flows (including imports and exports) clear slowly, a partial equilibrium 'asset block' may be used to determine exchange rates (or the balance of payments) at every point of time, whereas the current account is asserted to be affected by the exchange rate 'later on'.

The general view of dynamic processes underlying the moving equilibrium method is well expressed by Samuelson (1947, 330-331) as follows: 
I, myself, find it convenient to visualize economic processes of quite different speed, some very slow compared to others. Within each long run, there is a shorter run, and within each shorter run there is still a shorter run, and so forth in infinite regression. For analytical problems it is often convenient to treat slow processes as data and concentrate upon processes of interest. For example, in a short run study of the level of investment, income, and employment, it is often convenient to assume that the stock of capital is perfectly or sensibly fixed. ${ }^{1}$

In a similar vein, Hicks (1939, 122-123) implicitly invokes the Moving Equilibrium Theorem when writing:

Even if we decide to admit some small variability of output into our shortest period, nevertheless that shortest period (which I shall call a Week, to distinguish it from Marshall's Day) still needs to be clearly defined. I shall define a week as that period of time during which variations in price can be neglected. For theoretical purposes this means that prices will be supposed to change, not continuously, but in short intervals. The calendar length of the week is of course quite arbitrary; by taking it to be very short, our theoretical scheme can be fitted as closely as we like to that ceaseless oscillation which is as characteristic of prices in certain markets ....

A convenient way of visualizing this assumption of constant prices during the week is to suppose that there is only one day in the week (say Monday) when markets are open and contracts can be made. Contracts can, indeed, be carried out during the week (goods can be delivered, and so on); but no new contracts can be made until Monday week. Monday's prices will therefore rule during the week .... Now it is not hard to see that prices will remain constant during the week, when markets are not open, and then there is therefore no opportunity for prices to change. But we need also to try and bring ourselves to suppose that price-changes are negligible during market hours on the Monday, when the market is open and dealers have to fix market prices by higgling and bargaining, trial and error. This implies that the market (indeed all markets) proceeds quickly and smoothly to a position of temporary equilibrium -- in Marshall's sense.

Even modern formal treatments invoke the Moving Equilibrium Theorem without bothering to ask for a proof. Arrow and Hahn $(1971,49)$ write, for instance:

\footnotetext{
${ }^{1}$ It may be of interest to note that Samuelson's analytical treatment of the moving equilibrium method differs from what is suggested in this quotation. He views the method as a method of successive approximation involving the sequential determination of higher-order derivatives; see Samuelson (1947). The present treatment is inspired by Lotka (1957, Ch. 2).
} 
It is evidently both useful and legitimate to distinguish between an economy in which there is a full complement of futures markets ... and one that is not so fortunate. The equilibrium in the former economy might be called a 'full' or 'long-term' equilibrium, while the latter may be analyzed by means of a sequence of short-run equilibria.

Allen $(1967,79)$, in his discussion of economic dynamics, describes the method of equilibrium dynamics as follows: ${ }^{2}$

The simplest equilibrium dynamic system arises when the demand and supply schedules shift over time in some specified way: $D=D(P, t)$ and $S=S(P, t)$. An equilibrium path of $\bar{P}$ is obtained by solving $D(P, t)=S(P, t)$.

All this is presented as a matter of course, but it involves a logical problem. If price changes are governed by excess supply, then the assumption of supply equalling demand rules out any price changes. This has been stressed by Samuelson $(1947,323)$ who writes:

Paradoxically, on the assumption that the price is not changing, we derive a moving equilibrium path for the price!

The Moving Equilibrium Theorem states that this paradox is not to be seen as invalidating the moving equilibrium method.

\section{The Moving Equilibrium Method and Partial Analysis}

Partial analysis concentrates on a set of selected variables (like prices and quantities in a single market) while taking all other influences as fixed, and ignoring all possible repercussions through the economic system. The following example illustrates the relationship between partial analysis and the moving equilibrium method.

Consider a system of differential equations with two state vectors, $x$ and $y$, of "fast" variables, and one state vector $z$ of "slow" variables. Let us assume that the

\footnotetext{
2 In order to integrate the following example into the present framework, I interpret the time variable t as entering through intermediate variables, say $z=z(t)$, with $D=D(P, t)$ standing for $D=D(P, z(t))$, and so forth. The discussion of non-autonomous systems has been sidestepped here. It poses no problem in the linear case, however, since non-autonomous systems can be reduced to autonomous systems by considering the deviations of the state vector from some particular solution to the system as a new vector of variables.
} 
two sets of fast variables do not interact directly but only through their impact on the slow state variable. The vectors $x$ and $y$ may, for example, describe the states of two unrelated markets that interact only through global variables $z$ like income, or exchange rates:

$$
\dot{x}=f(x, z), \quad \dot{y}=g(y, z), \quad \dot{z}=h(x, y, z) .
$$

The system can be analyzed by using the moving equilibrium method. The moving equilibria $X(z)$ and $Y(z)$ ) can be determined and plugged into the equation describing the movement of the slow variable. This yields $\dot{Z}=$ $h(X(Z), Y(Z), Z)$. If $x$ and $y$ are indeed sufficiently fast and damped, then the stability conclusions reached in this way will be correct. If this were not the case, then the dynamic analysis of the partial systems $f$ or $g$ would be of doubtful validity, as there would be no reason to suppose that the results carry over to the full system.

This point may even be strengthened by pointing out that any given system may be conceived as embedded in a still larger system, and all conclusions we may reach refer, as a matter of principle, to partial systems. It is thus important to have reasons for believing that the conclusions reached in partial models remain valid in a more comprehensive setting.

\section{Generalizing the Moving Equilibrium Method}

The proof of the Moving Equilibrium Theorem has used the fact that any eigenvalue $\lambda_{i}(\alpha)$ of $J(\alpha)$ approaches either an eigenvalue of $\left(D-C A^{-1} B\right)$ or has the property that $\frac{\lambda_{i}(\alpha)}{\alpha}$ approaches an eigenvalue of A. Since there are exactly $m+n$ eigenvalues to $J, m$ eigenvalues to $A$ and $n$ eigenvalues to $\left(D-C A^{-1} B\right)$, the eigenvalues of the fast system (4) and of the reduced system (8) taken together characterize the spectrum of the true system in the limit, and $k$ equals $m$. In this sense, the moving equilibrium method is not confined to stable systems, but is applicable in the more general sense of yielding correct dynamic conclusions.

Furthermore, the method may be viewed as an aggregation procedure in that it reduces dimensionality: the full system (4), (5) operates in $m+n$ dimensions, but the reduced system (8) operates only in $n$ dimensions. This thought may be generalized to yield a fully-fledged aggregation method in the linear case (Schlicht, 1990, 1992). 
There also exists a non-linear version of the Moving Equilibrium Theorem, but this relies on the existence of certain Ljapunov functions which restrict its scope (Schlicht, 1985, 39-44).

\section{Acknowledgement}

I thank Andreas Nicolin for helpful comments.

\section{References}

Allen, R. G. D. (1967). Macro-economic theory - a mathematical treatment. New York: Macmillan.

Arrow, K. J., and Hahn, F. H. (1971). General competitive analysis. Edinburgh: Oliver \& Boyd. URL https://openlibrary.org/works/OL10551730W/General_ competitive_analysis.

Eckalbar, J. C. (1985). On the use and misuse of the instantaneous speed of adjustment assumption. Economic Modelling, 2(1): 3 - 7. ISSN 0264-9993. DOI http://dx.doi.org/10.1016/0264-9993(85)90003-3.

Hicks, S. J. R. (1939). Value and Capital - An Inquiry Into Some Fundamental Principles of Economic Theory. Oxford: Clarendon Press. URL https: //openlibrary.org/books/OL6387641M/Value_and_capital.

Lindbeck, A. (1976). Approaches to Exchange Rate Analysis-An Introduction. Scandinavian Journal of Economics, 78(2): 133-45. URL http://econpapers. repec.org/RePEc:bla:scandj:v:78:y:1976:i:2:p:133-45.

Lotka, A. J. (1957). Elements of mathematical biology. New York: Dover. URL https://openlibrary.org/books/OL23807423M/Elements_of_mathematical_ biology. First published in 1925.

Marshall, A. (1920). Principles of Economics. London: Macmillan, 8 edition. URL http://www.econlib.org/library/Marshall/marPtoc.html. First edition 1890,.

Samuelson, P. A. (1947). Foundations of economic analysis. Cambridge (MA): Harvard University Press. URL https://openlibrary.org/books/OL13661872M/ Foundations_of_economic_analysis. 
Schlicht, E. (1978). Die Methode der Gleichgewichtsbewegung als Approximationsverfahren. In E. Helmstädter (Ed.), Neuere Entwicklungen in den Wirtschaftswissenschaften, pages 293-305. Berlin: Duncker \& Humblot. URL https://epub.ub.uni-muenchen.de/3149/.

Schlicht, E. (1985). Isolation and Aggregation in Economics. Berlin-HeidelbergNew York-Tokyo: Springer-Verlag. URL http://EconPapers.repec.org/RePEc:lmu: muenbk:3.

Schlicht, E. (1990). Local Aggregation in a Dynamic Setting. Journal of Economics, 51(3): 287-305. URL https://epub.ub.uni-muenchen.de/2118/.

Schlicht, E. (1992). Marshall, Keynes, and Macroeconomics. Quaderni di Storia dell'Economia Politica, (1): 511-529. URL https://epub.ub.uni-muenchen.de/ $11055 /$.

Schlicht, E. (1997). The moving equilibrium theorem again. Economic Modelling, 14(2): 271-278. URL http://econpapers.repec.org/article/eeeecmode/v_3a14_ 3ay_3a1997_3ai_3a2_3ap_3a271-278.htm. 